\title{
DETECTION OF MYCOBACTERIAL ANTIGEN AND ANTIBODIES IN THE CEREBROSPINAL FLUID OF PATIENTS WITH TUBERCULOUS MENINGITIS
}

\author{
A. Chandramuki, P. R. J. Allen, ${ }^{*}$ M. Keen and J. Ivanyi $\dagger$ \\ Department of Experimental Immunobiology, The Wellcome Research Laboratories and \\ the * Department of Immunotechnology, Wellcome Biotechnology Ltd, Beckenham, Kent
}

\begin{abstract}
Summary. An immunodiagnostic test for the detection of a soluble nonprotein mycobacterial antigen by reverse passive haemagglutination with IgM murine monoclonal antibody was developed. The test was used to analyse the cerebrospinal fluid of 89 patients with tuberculous meningitis (TBM) from India and 127 control subjects from India and the UK. The antigen was demonstrable in $88 \%$ of culture-positive and $73 \%$ of culture-negative TBM patients. However, it was also detected in $21 \%$ of Indian patients with pyogenic meningitis, and in $8 \%$ of Indian and $1 \%$ of UK control subjects. Antibodies binding to a soluble mycobacterial extract were detected at low titre in $68 \%$ of all subjects with TBM and in $37 \%$ of Indian cases of pyogenic meningitis. Because patients with TBM had raised levels of the antigen and of antibodies to the antigen, the possible role of immune complexes in the pathogenesis of the disease is briefly discussed.
\end{abstract}

\section{INTRODUCTION}

Tuberculous meningitis (TBM) is one of the most common forms of extrapulmonary tuberculosis in the Indian subcontinent and represented, in the past decade, almost one third of all cases of tuberculosis admitted to a children's hospital in Bombay (Udani, 1980). Although TBM is rare in advanced countries, its importance is exemplified by a report that between 1960 and 1970 TBM accounted for $48 \%$ of cases of bacterial meningitis in one major centre in Scotland (Kennedy and Fallon, 1979). Rapid diagnosis of TBM is essential if mortality and permanent morbidity are to be reduced. Unfortunately, Ziehl-Neelsen staining of cerebrospinal fluid (CSF) reveals bacilli in only a fraction of cases subsequently confirmed by culture. A particular problem for clinicians is the early distinction between TBM and partially-treated "pyogenic" bacterial meningitis.

As an alternative to bacteriological examination, antibodies agglutinating red blood cells coated with purified protein derivative (PPD) were detected in the concentrated CSF of most TBM patients but not of control subjects (Kuo, 1969;

Received 2 Nov. 1984; accepted 29 Jan. 1985.

$\dagger$ Requests for reprints should be sent to J. Ivanyi, MRC Tuberculosis and Related Infections Unit, Royal Postgraduate Medical School, Du Cane Road, London W12 0HS. 
Munoz et al., 1978). Recently, mycobacterial antigens were detected in the CSF from TBM patients by a "tandem" immunoenzymatic assay, in which a commercial rabbit anti-BCG globulin was used to coat the solid-phase and as the enzyme-labelled reagent (Sada et al., 1983).

The development of specific and sensitive immunoassays of either antigen or antibody may benefit from monoclonal antibodies (MABs). An MAB of IgM isotype (ML34) reacting with Mycobacterium tuberculosis, $M$. leprae and $M$. bovis, and with other species of mycobacteria, appeared promising for the sensitive detection of a soluble nonprotein cell-wall antigen which was designated MY4 (Ivanyi et al., 1983 and in press; Praputpittaya and Ivanyi, 1985). In this paper, ML34 antibody coupled to sheep red-blood cells (SRBC) was used for the detection of mycobacterial antigen or immune complexes in CSF by a reverse passive haemagglutination assay (RPHA). Furthermore, antibodies to mycobacterial antigens were determined by solid-phase radioimmunoassay (SPRIA), the antibody competition test (ACT) (Hewitt et al., 1982; Ivanyi et al., 1983) and the immunoblot technique.

\section{MATERIALS AND METHODS}

Patients. Group IA was represented by patients from South India with a clinical diagnosis of TBM confirmed by culture of tubercle bacilli from the CSF. Patients with the same clinical diagnosis but unconfirmed by culture formed group IB. Group IIA comprised Indian patients with a clinical diagnosis of pyogenic meningitis and bacteriological evidence of gram-positive cocci or gram-negative bacilli. Group IIB represented cases without symptoms of meningitis; most had head injury or mental retardation.

Group IIIA comprised UK patients with viral meningitis or neurosyphilis. Group IIIB included patients with multiple sclerosis, demyelinating diseases or cervical spondylosis.

$C S F$ samples were centrifuged within $1 \mathrm{~h}$ of delivery for $10 \mathrm{~min}$ at $3000 \mathrm{rpm}$, stored at $-20^{\circ} \mathrm{C}$, absorbed with uncoated SRBC and used neat and at serial dilutions in phosphate buffered saline (PBS).

Detection of MY4 antigen by reverse passive haemagglutination. The globulin fraction precipitated from ascitic fluid containing the murine IgM monoclonal antibody ML34 (Ivanyi $e t$ al., 1983) by $18 \%$ sodium sulphate was coupled to chymotrypsin $(5 \mathrm{mg} / \mathrm{ml}$, Sigma)-treated SRBC by treatment with chromic chloride (Scott et al., 1981). Briefly, $50 \mu \mathrm{l}$ of chromic chloride $0.033 \%$ was added by drops to a mixture of $25 \mu \mathrm{l}$ of ML34 globulin $(400 \mu \mathrm{g} / \mathrm{ml})$ and $25 \mu \mathrm{l}$ of packed SR BC; this suspension was incubated on a mixer for $1 \mathrm{~h}$ at $20^{\circ} \mathrm{C}$. The cells were washed and made up to a $1 \%$ suspension in PBS. Coupled erythrocytes were stored at $4^{\circ} \mathrm{C}$ and discarded after 1 week. Doubling dilutions of CSF were made in U-shaped microtitration plates (Dynatech) in $25-\mu \mathrm{l}$ volumes. ML34-coated red cells $(25 \mu \mathrm{l})$ were added and haemagglutination reactions were read after incubation for $1 \mathrm{~h}$ at $20^{\circ} \mathrm{C}$ and overnight at $4^{\circ} \mathrm{C}$. Controls included neat CSF samples with uncoated SRBC, and PBS with coated SRBC. Furthermore, satisfactory negative results were obtained with SRBC coated with an IgM MAB of unrelated specificity (human TSH). The ML34-coated SRBC were agglutinated by a soluble extract (Ivanyi et al., in press) from $M$. tuberculosis (MTSE) as a positive control, but not by other ML34-negative species of mycobacteria (Ivanyi et al., 1983) such as $M$. xenopi, M. scrofulaceum or $M$. nonchromogenicum. Haemagglutination occurred in the presence of MTSE $1.0 \mu \mathrm{g} / \mathrm{ml}$.

Detection of antibodies to soluble mycobacterial antigens by solid-phase radioimmunoassay (SPRIA) (Ivanyi et al., 1983 and in press). Microtitration plates coated with MTSE were incubated with four serial doubling dilutions of CSF overnight at $4^{\circ} \mathrm{C}$. Plates were washed and incubated with ${ }^{125}$ I-labelled rabbit anti-human Ig (RaHG) $(50000 \mathrm{cpm}$ well). Bound radioactivity was expressed as a relative value, $100 \%=$ binding to human Ig-coated wells; $0 \%=$ background binding to bovine serum albumin-blocked wells. Antibody levels were expressed as reciprocal dilutions of CSF that gave $50 \%$ binding (ABT50). 
Antibody competition test (ACT) for the detection of MAB-like epitope-specific antibodies in CSF (Hewitt et al., 1982; Ivanyi et al., 1983). ${ }^{125}$ I-labelled probes were prepared of monoclonal antibodies ML34, TB72, ML30, TB23, TB68, TB78 and TB71. Competitive inhibition of the binding of each of the MABs to MTSE-coated microtitration plates by the presence of CSF samples was measured. The results were expressed as relative inhibition ( $\%$ ) of ${ }^{125} \mathrm{I}-\mathrm{MAB}$ binding.

Immunoblot analysis of antibodies from CSF. MTSE antigen was separated by electrophoresis in SDS- $15 \%$ polyacrylamide gels and the bands were electroblotted on to nitrocellulose sheets by previously used techniques (Ivanyi et al., 1983). Nitrocellulose strips were incubated with CSF samples then with ${ }^{125}$ I-RaHG and the radioactive blots developed by autoradiography.

\section{RESULTS}

The MY4 antigen was demonstrated in the CSF of 22 out of 25 culture-positive and in 47 of 64 culture-negative TBM patients (fig. 1; table I). MY4 titres were evenly distributed in the range 2-512 and false-negative values were found in only $12 \%$ of the culture-positive and $26 \%$ of the culture-negative groups.

In the Indian control groups, 4 out of 19 cases of pyogenic meningitis and one out of 13 subjects without clinical symptoms of meningitis were MY4 positive. A retrospective review of medical records failed to reveal any clinical or laboratory evidence of past tuberculosis in these patients.

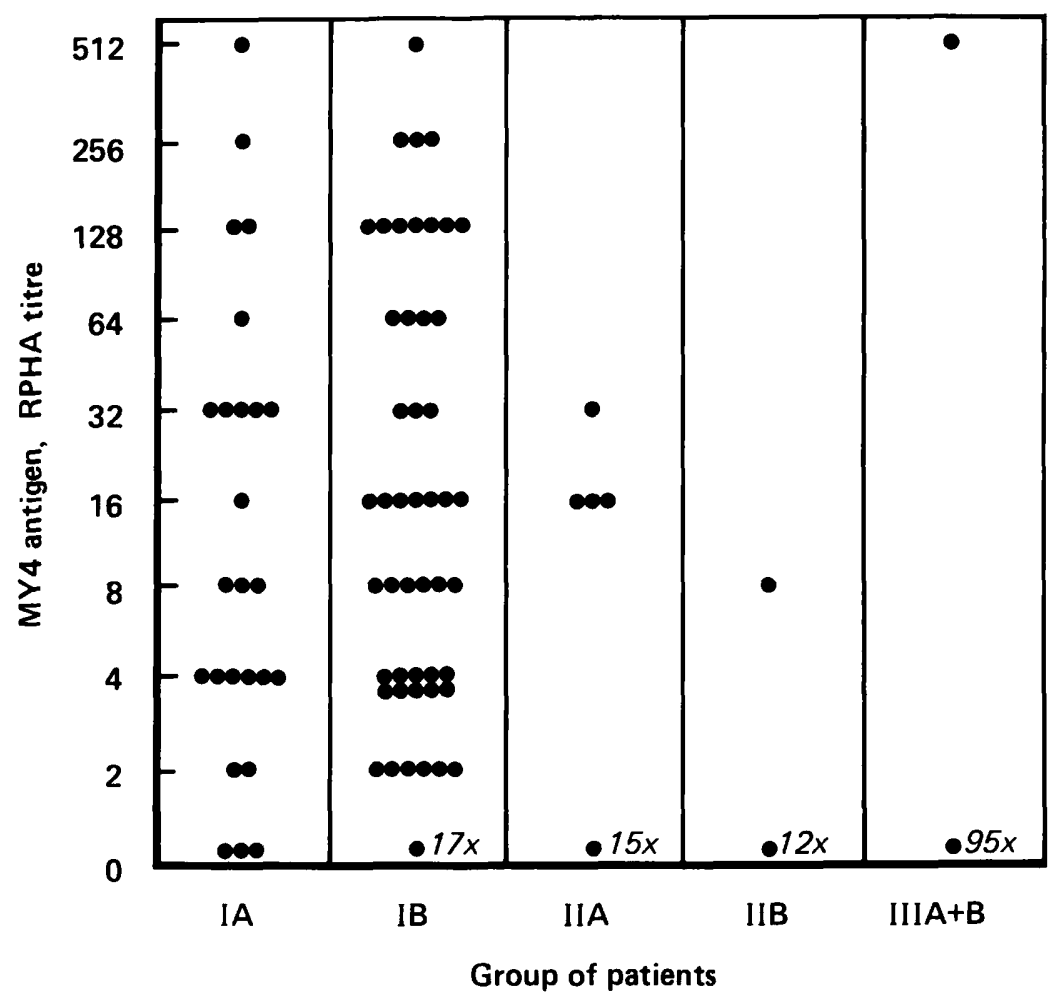

Fig. 1.-MY4 antigen levels in the CSF of patients with TBM and in control subjects. Symbols represent individual dilutions of CSF that agglutinated ML34-coated SRBC. For the designation of groups, see table I. 


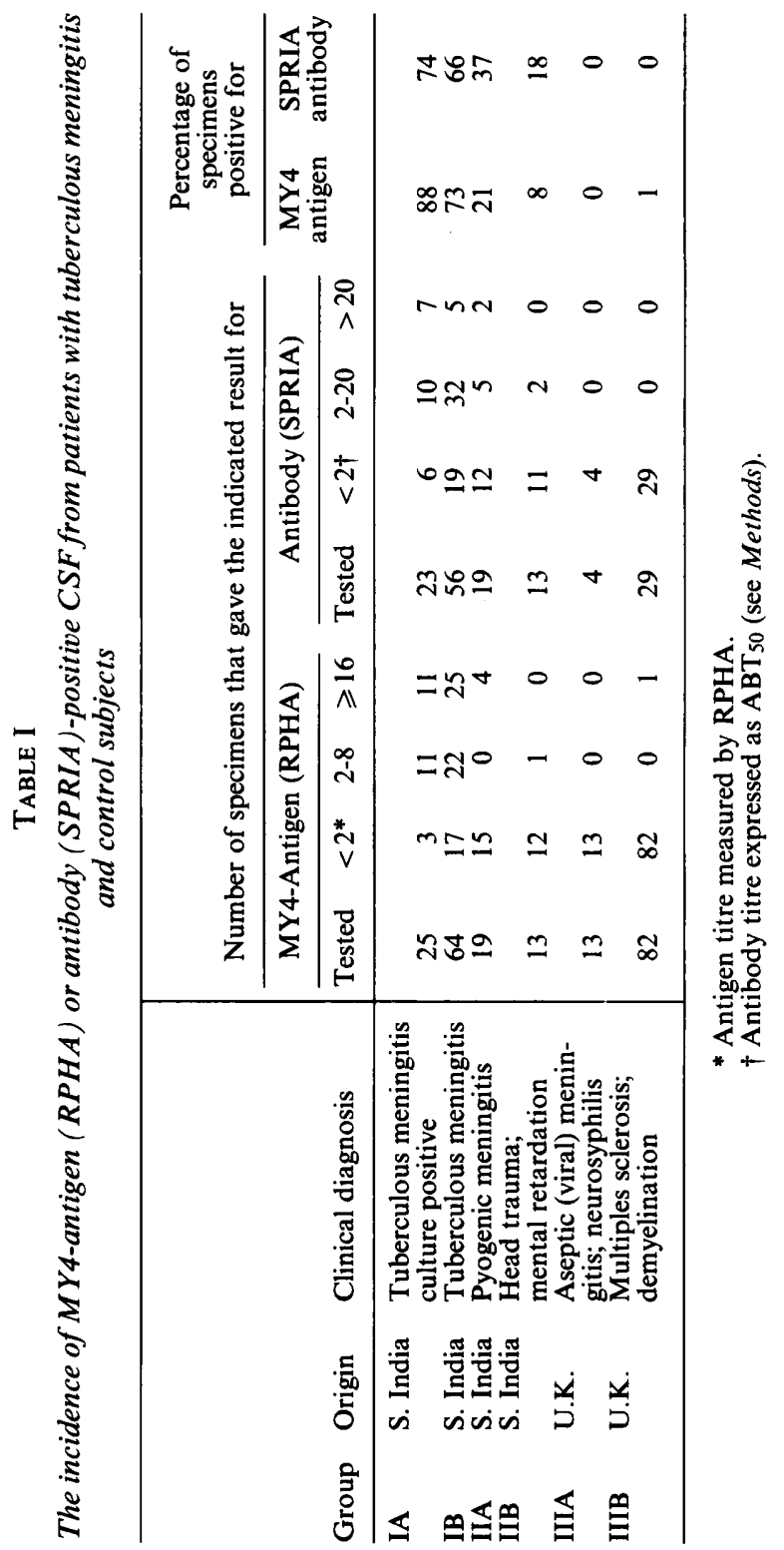


Of the 95 UK controls, a high level of MY4 antigen was found only in one 40-year-old caucasian female with raised intracranial pressure and enlarged ventricles; she was not accessible for follow-up study.

Antibodies binding to MTSE were detected by the SPRIA technique in $68 \%$ of all TBM patients (fig. 2; table I). Antibodies, mostly at low levels, were found in CSF from $37 \%$ of Indian patients with pyogenic meningitis, in $18 \%$ of Indian controls without meningitis, but in none of the UK controls.

These data were analysed for a possible correlation between MY4-antigen and SPRIA-antibody levels; furthermore, the specificity of antibodies was investigated by ACT and immunoblot assays (table II). The results failed to show any correlation between MY4-antigen (RPHA) and -antibody (SPRIA) levels: individual patients had either antigen alone, antibody or antigen-antibody complexes. The results of antibody competition tests with 32 CSF samples from patients with TBM showed a positive correlation between SPRIA binding titres and ML34-inhibitory activity but failed to show any correlation with the TB72 antibody probe. The immunoblot analysis of eight CSF samples showed bands restricted to the $(30-40) \times 10^{3}-\mathrm{mol}$. wt region, which corresponded with the binding of ML34 antibody with the MY4 antigen (Ivanyi et al., 1983 and in press) (fig. 3). The intensity of immunoblotted bands apparently correlated

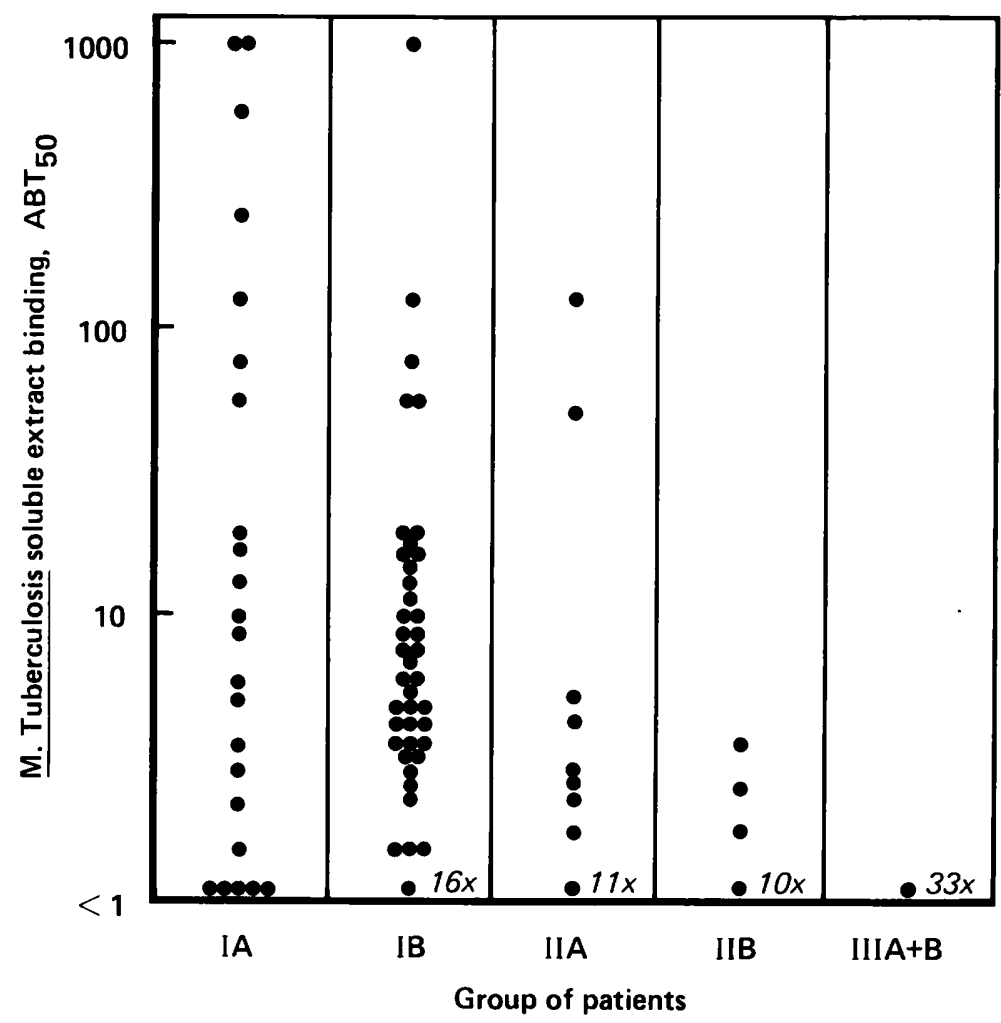

FIG. 2.-Antibody levels in the CSF of patients with TBM and in control subjects. Symbols represent individual dilutions of CSF that gave $50 \%$ binding in the SPRIA. For the designation of groups, see table I. 


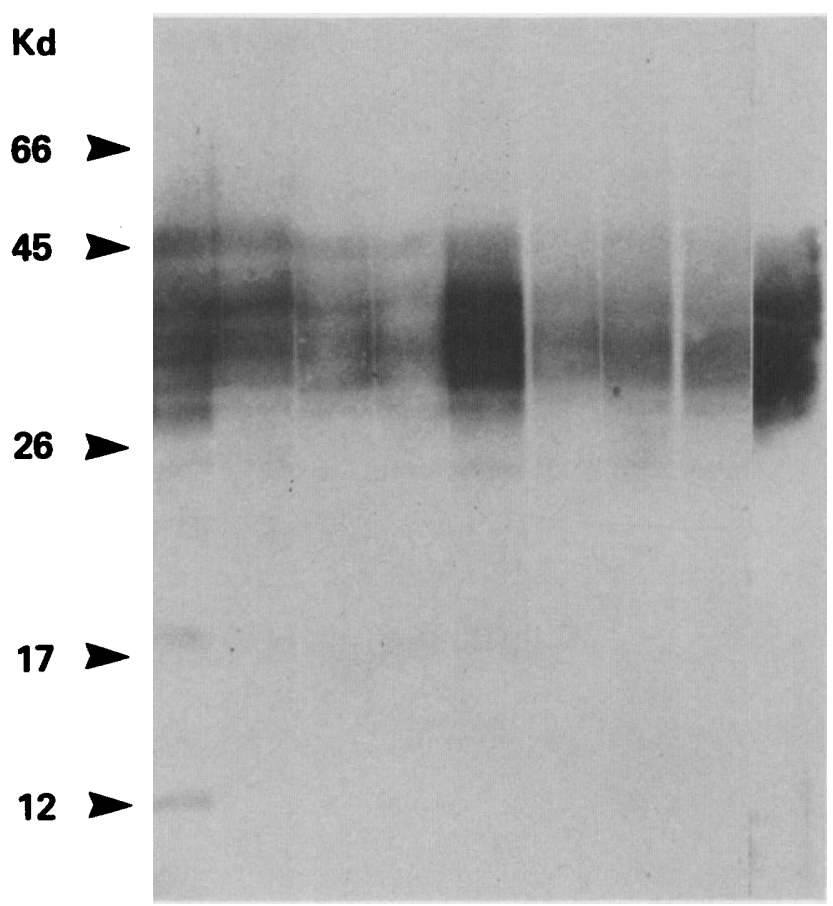

\section{$\begin{array}{llllllllll}\text { CSF No.: } & 1 & 2 & 3 & 4 & 5 & 6 & 7 & 8 & \mathrm{ML} \\ \end{array}$ \\ $\begin{array}{lllllllll}\text { ML34 competition (\%): } & 66 & 34 & 14 & 10 & 84 & 10 & 11 & 15\end{array}$

$\begin{array}{lllllllll}\text { Log }_{2} \text { RPHA titre: } & 1 & 0 & 0 & 5 & 8 & 7 & 5 & 2\end{array}$

FIG. 3.-Immunoblot analysis of antibodies in the CSF. Nitrocellulose strips, carrying SDS-PAGE-separated $M$. tuberculosis antigens, were treated with eight CSF samples. ML34 antibody was included as a control.

with the ML34-inhibitory activity of CSF samples. Sample no. 5 contained high levels of MY4 antigen as well as antibody.

None of the TBM cases showed significant competition by the CSF with the binding of the other ${ }^{125}$ I-labelled MABs-ML30, TB23, TB68, TB78 and TB71. However, it is of interest to note that ML30-inhibitory activity, stronger than that towards ML34 and TB72, was observed in two Indian patients with pyogenic meningitis (results not shown).

\section{DiscUSSION}

Demonstration of the mycobacterial MY4 antigen in CSF specimens from patients with TBM has implications for the specific immunodiagnosis of the disease. The main attribute of the reverse passive haemagglutination test is its speed, which is highly desirable for avoiding any delay in the institution of antituberculosis therapy. High sensitivity of the assay allows the testing of CSF without the need for a delaying and 


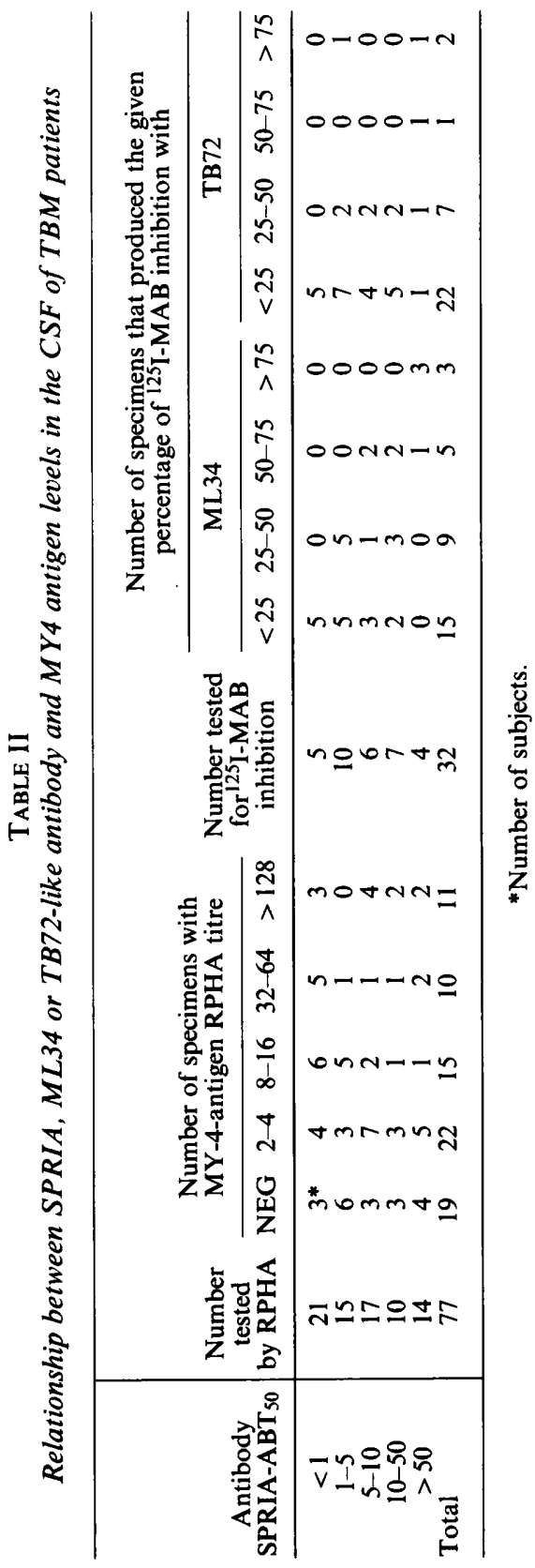


cumbersome concentration of the fluid. Moreover, the simple performance and visual reading of haemagglutination makes the assay amenable for routine performance in any basic clinical laboratory.

The MY4 antigen may have been shed into the CSF by $M$. tuberculosis in vivo, because disintegration of bacilli seemed unlikely to occur during the handling or storage of the CSF. Previous studies indicated that the MY4 antigen is a subtilisinresistant molecule, possibly of highly glycosylated structure (Ivanyi et al., 1983 and in press; Praputpittaya and Ivanyi, 1985). The IgM isotype of the ML34 antibody and the repeating nature of the corresponding MY4b determinant probably contributed to the sensitivity of detection by RPHA. The antigen is present in the mycobacterial cell-wall fraction and expressed on the surface of $M$. tuberculosis but has an inside orientation in M. leprae (Praputpittaya and Ivanyi, 1985).

Analysis of the specificity of antibodies in CSF by the immunoblot and competition assays indicated that the antibodies are mainly directed towards the MY4 antigen, because inhibition of binding of TB72 antibody was low and competition with several other MABs could not be detected. The lack of competition with ML30 in TBM patients was particularly surprising, considering that this MAB is directed towards the main protein constituent of PPD (Ivanyi et al., in press), towards which antibodies (Kuo, 1969; Munoz et al., 1978), and poliferative responses by CSF lymphocytes (Kinnman et al., 1981) have been reported.

Control subjects from the UK (with one exception) gave negative results in tests for antigen and antibody. Although the finding that 4 out of 19 cases of pyogenic meningitis and one out of 13 controls without meningitis from India gave positive results in tests for MY4 antigen may limit the specificity of the RPHA test, the interpretation of these findings remains conjectural without further clinical and laboratory information about the patients. In particular, concomitant tuberculous lesions in patients from groups IIA and IIB were not recorded clinically but could not be excluded on the basis of CSF culture. Alternatively, we considered the possible contribution of MY4 antigen derived from various "atypical" and environmental mycobacteria which react with ML34 antibody (Ivanyi et al., in press). MY4 antigen, free or in immune complexes (Carr et al., 1980), could have entered the CSF from the circulation through a blood-brain barrier impaired by the inflammatory disease. This interpretation could be verified by analysis of sera in future studies. Finally, cross-reactions between antigens from mycobacteria and those from other bacterial genera have been described (Minden et al., 1972), but do not occur with the MY4 epitope because several members of the genera Corynebacterium, Propionibacterium, Nocardia, Streptomyces, Actinomyces, Neisseria, Streptococcus and Haemophilus failed to react with ML34 by SPRIA (results not presented).

The reported data demonstrate a considerable degree of individual variation in CSF concentrations of MY4 antigen, antibody and their complexes. Further studies are needed to ascertain a possible association between the occurrence of complexes and clinical symptoms. It was suggested that the release of antigens from tubercle bacilli is primarily responsible for initiating the pathological changes of TBM in previously sensitised subjects (Udani, 1980). Thus, antigen-antibody complexes together with cell-mediated reactions would result in vasculitis, increased permeability of capillaries and, consequently, in brain oedema. This would also explain the resistance to antituberculosis drugs and the progressive nature of meningitis in patients at an 
advanced stage of the disease. Monitoring of MY4-antigen and antibody levels may help to determine when and how corticosteroid therapy, which aims mainly to reduce the cerebral oedema (Kennedy and Fallon, 1979), should be used.

The MY4-RPHA test cannot be directly related to the smear or culture test for tubercle bacilli, because MY4 levels would be determined by the rate of antigen shedding from live bacilli and its stability in CSF as well as the bacterial count. These aspects will need to be investigated also when considering the application of RPHA technique for other body fluids and secretions.

We thank Professor I. C. McLennan, Department of Immunology, University of Birmingham, and staff of the Queen Elizabeth Hospital, Birmingham, for providing CSF samples for analysis; we thank also members of the Department of Microbiology, NIMHANS, Bangalore, for the dispatch of biological samples.

AC was a British Council Commonwealth Fellow visiting the UK from the Department of Microbiology, National Institute of Mental Health and Neurosciences, Bangalore, India.

\section{REFERENCES}

Carr R I et al., 1980 Immune complexes and antibodies to BCG in sera from patients with mycobacterial infections. Clinical and Experimental Immunology 39:562-569.

Hewitt J, Coates A R M, Mitchison D A, Ivanyi J 1982 The use of murine monoclonal antibodies without purification of antigen in the serodiagnosis of tuberculosis. Journal of Immunological Methods 55:205-211.

Ivanyi J, Krambovitis E, Keen M 1983 Evaluation of a monoclonal antibody (TB72) based serological test for tuberculosis. Clinical and Experimental Immunology 54:337-345.

Ivanyi J, Sinha S, Aston R, Cussell D, Keen M, Sengupta U 1983 Definition of species specific and cross-reactive antigenic determinants of Mycobacterium leprae using monoclonal antibodies. Clinical and Experimental Immunology 52:528-536.

Ivanyi J, Morris J A, Keen M Studies with monoclonal antibodies to mycobacteria. In: Macario, AC, Macario AJL (eds) Monoclonal antibodies against bacteria. Academic Press, New York, in press.

Kennedy D H, Fallon R J 1979 Tuberculous meningitis. Journal of the American Medical Association 241:264-268.

Kinnman J, Fryden A, Eriksson S, Moller E, Link H 1981 Tuberculous meningitis: immune reactions within the central nervous system. Scandinavian Journal of Immunology 13:289-296.

Kuo C T 1969 The diagnosis of tuberculous meningitis by immunologic reaction of cerebrospinal fluid. American Review of Respiratory Diseases 100:565-568.

Minden P, McClatchy J K, Cooper R, Bardana E J, Farr R S 1972 Shared antigens between mycobacterium bovis (BCG) and other bacterial species. Science 176:57-58.

Munoz O, Guiscafre H, Feria A, Lopez M, Martinez-Cairo S 1978 Indirect hemagglutination with PPD in cerebrospinal fluid for the tuberculous meningoencephalitis. Archivos de Investigacion Medica (Mexico) 9:469-476.

Praputpittaya K, Ivanyi J 1985 Detection of an antigen (MY4) common to M. tuberculosis and M. leprae by 'tandem' immunoassay. Journal of Immunological Methods 79:149-157.

Sada E, Ruiz-Palacios G M, Lopez-Vidal Y, Ponce de Leon S 1983 Detection of mycobacterial antigens in cerebrospinal fluid of patients with tuberculous meningitis by enzyme-linked immunosorbent assay. Lancet 2:651-652.

Scott M L, Thornley M J, Coombs R R A, Bradwell A R 1981 Measurement of human serum IgE and IgA by reverse passive antiglobulin haemagglutination. International Archives of Allergy and Applied Immunology 64:222-229.

Udani P M 1980 Tuberculosis in children with special reference to neurotuberculosis. Annals of the National Academy of Medical Sciences (India) 16:121-161. 